\section{Genetic Insights from a Molecular Pathway Analysis on Two Independent Samples of Autistic Patients}

\author{
Drago $\mathrm{A}^{1 *}$, Calabro $\mathrm{M}^{2}$, Crisafulli $\mathrm{C}^{2}$ and Rasmussen $\mathrm{SK}^{3}$
}

${ }^{1}$ Department of Clinical Medicine, Aarhus University, Psykiatrisk Forskningsenhed Vest, Herning, Denmark

${ }^{2}$ Department of Biomedical Science and Morphological and Functional Images, University of Messina, Via Consolare Valeria, 98125 Messina, Italy

${ }^{3}$ Via University College, University in Horsen, Denmark

\section{Abstract}

Background: Autism spectrum disorder (ASD) is a neurodevelopmental disorder characterized by limited interest and lacking ability in social interactions, repetitive behavior and dysfunction in social communication. ASD runs in families. Twin studies suggest a strong genetic basis for ASD. The complete definition of a genetic profile at risk for ASD is nevertheless currently lacking.
\end{abstract}

Methods: NIHM-Autism datasets 3 and 4 ( $n=1233$ and $n=2890$ respectively) were analyzed. A molecular pathway analysis was conducted. Quality analysis was run as usual ( $\lambda$ values). Plink and R (ReactomePA and Bioconductor packages) served for TDT, association tests and the molecular pathway analysis.

Results and Discussion: The "Adherens junctions' interactions pathway" and "Axon guidance" were enriched in the first sample, while the "Extracellular matrix organization pathway" was enriched in the second sample. The "Axon guidance pathway" showed a trend for enrichment in the second sample. A trend of significant enrichment was observed for the "NCAM1 molecular pathway" when the severity of autistic symptoms was investigated.

Conclusion: Cell to cell interaction and the cell-matrix interaction may hold the genetic risk for ASD. Both neurodevelopment and immune response (T-cell) rely on those processes and may be involved in the pathophysiology of ASD.

Keywords: Autism; Genome-Wide Association Study (GWAS); Molecular pathway analysis; Enrichment

\section{Introduction}

Autism spectrum disorder (ASD) is a neurodevelopmental disorder that appears early in life and is characterized by limited interest and lacking ability in social interactions, repetitive behavior and dysfunction in social communication. Motor and intellectual deficits, together with mood and sleep disorder and sensory and gastrointestinal abnormalities are common [1]. ASD affects up to $1 \%$ of the general population [2] and has a genetic basis $[3,4]$. A number of genetic variations have been associated with ASD (https://gene.sfari.org/autdb/GS_Home. do), but results are not definitive [5-11]. A notable exception was recently published [12], reporting a positive association finding for a couple of genes previously associated with Autism, the SHANK3 and the WBSCR17. Apart from this result, the overall lack of replication rate in genetic association finding is in conflict with evidence showing that the heritability of ASD due to common variants is as high as $60 \%$ [13]. Limited power of the analysis, small sample sizes, and a critical phenotype definition may be limitations of the studies conducted so far [14-16]. A possible solution to this sparse genetic evidence is the "many genes common pathways" hypothesis, which suggests that the impact of different genes may converge to common pathways, whose impaired function results in the core symptoms of a disease [17]. The molecular pathways analysis is consistent with this hypothesis and may help understanding complex genetic diseases [18].

Previous results about the genetic networks whose disruption led to ASD showed that the following molecular pathways might be involved: Protein synthesis and metabolism, modulation of transcription process, chromatin remodeling, calcium signaling and the oxytocin pathway [19-21]. Nevertheless, those findings were generated by systematic reviews, and to the best of our knowledge a molecular pathway analysis on GWAS data derived from ASD trios is yet to be conducted.

In the present contribution two independent trios sample of ASD individuals are retrieved from the NIMH database (https://www. nimhgenetics.org/). A molecular pathway analysis is conducted on the transmission disequilibrium test (TDT) result in order to identify common molecular pathways enriched in variations associated with Autism in both samples. Moreover, the same approach is used to identify one or more pathways enriched in variations associated with the severity of autistic symptoms as ranked from 1 to 4 (from broad spectrum to strict Autism) in one of the samples. The present contribution is originated from a previous published abstract at the $29^{\text {th }}$ ECNP Conference [22].

\section{Materials and Methods}

\section{Dataset}

Genetic data were available from the NIMH genetics (https://www. nimhgenetics.org/). The Autism Dataset 4 (Study 65/TASC GWAS Data) sample was chosen for the investigation sample. The Autism Dataset 3 (GWAS Data on 1,264 Non-AGRE Samples) served as a replication sample. GWAS were conducted with Affymetrix 5.0 in both samples.

\section{Genetic analysis and quality control}

SNPs (Single Nucleotide Polymorphism) were excluded for allele

*Corresponding author: Dr. Antonio Drago, Department of Clinical Medicine Aarhus University, Psykiatrisk Forskningsenhed Vest, 7400 Herning, Denmark, Tel: +45 784 74660; E-mail: antonio.drago76@gmail.com

Received February 05, 2017; Accepted February 09 2018; Published February 13, 2018

Citation: Drago A, Calabro M, Crisafulli C, Rasmussen SK (2018) Genetic Insights from a Molecular Pathway Analysis on Two Independent Samples of Autistic Patients. J Mol Genet Med 12: 326 doi:10.4172/1747-0862.1000326

Copyright: ( 2018 Drago A, et al. This is an open-access article distributed unde the terms of the Creative Commons Attribution License, which permits unrestricted use, distribution, and reproduction in any medium, provided the original author and source are credited 
frequency $<0.01$ and low genotype call rate. Mendellian error threshold was set at $5 \%$ for families and $10 \%$ for single variations and missing data rate was set at $5 \%$ for the Dataset. Duplicate samples, excessive mendellian errors and monozygous twin (removed one of the twins) were sample exclusion criteria. Deviations from the Hardy-Weinberg equilibrium were accepted under a P-threshold of 0.0001 . Lambda values served to exclude inflation factors.

\section{Statistical analysis}

In the principal analysis, a transmission disequilibrium test (TDT) [23] was used to test for the presence of a genetic linkage between each and every SNP passing the quality test and the phenotype under analysis. The samples are comprised of trios were parents are labeled as " 0 " (cases) and the ASD child or children are labeled as " 2 " (controls). For the secondary analysis, an association analysis for quantitative data (command -assoc in plink) was run for every and single SNP using the phenotype from 1 to 4 available from the NIMH database, indicating different degrees of severity of autistic symptoms.

\section{Enrichment analysis}

Plink [24] served for the TDI GWAS analysis and genetic annotations. R [25] and dedicated packages served for the analyses. SNPs associated with the phenotype under analysis at a $p$ level $<0.05$ were included in the molecular pathway analysis. The enrichment analysis was conducted in an R environment [25], through Bioconductor [26] and the package ReactomePA [27]. The Reactome [28] is a manually curated database that includes chemical reactions, biological processes and molecular pathways. Reactome PA was developed to analyze molecular pathways associations with gene lists obtained from highthroughput genomic investigations. Bonferroni, and False Discovery rate q-values are incorporated for multiple comparison corrections.

\section{Results}

1233 individuals were available from the Autism Dataset 3, 789 males, 444 females and 588 cases. 333 nuclear families, 3 founder singletons were detected. 579 non-founders with 2 parents in 321 nuclear families, 14 non-founders without 2 parents in 9 nuclear families and 579 affected offspring trios were detected. 393763 markers were included in the analysis. Genomic inflation was excluded $(\lambda=1.03)$. The "Adherens junctions interactions pathway" was significantly enriched ( $p=0.000008$; adj. $p=0.008$ ) (Table 1). 2890 individuals (1824 males, 1069 females) with non-missing phenotype were found in the Autism Dataset 4, 936 cases, 1954 controls and 3 missing. 1930 founders and 963 non-founders were found, the total genotyping rate was 0.93 .965 nuclear families were detected, 2 founder singletons were found and 963 non-founders with 2 parents in 963 nuclear families were included in the analysis. 934 affected offspring trios were identified. 1160305 markers were available for the analysis. Genomic inflation was excluded $(\lambda=1.002)$. The "Extracellular matrix organization pathway" was significantly enriched ( $p=0.000008$; adj. $\mathrm{p}=0.007)$ in this sample. The "Axon guidance pathway" showed a trend for enrichment $(p=0.00019$; adj.p=0.084). A trend of significant enrichment was observed for the "NCAM1 molecular pathway" when the severity of autistic symptoms was investigated ( $p=0.00009$; adj.p $=0.097)$ (Table 2). Finally, the "NCAM1 molecular pathway" showed a trend for association with a worse presentation of the autistic symptoms when only subjects with autism were selected out of the total sample ( $p=0.00009$; adj. $p=0.097)$.

\section{Discussion}

ASD is a frequent condition in the general population, characterized by impaired social abilities, restricted interests and repetitive behavior. The disorder was consistently proven to have a genetic basis, but the number of involved genes could be as high as hundreds, which suggests a polygenic nature. In order to test the convergence of specific molecular pathways towards ADS, a metabolic pathway analysis was undertaken in two independent samples of Autistic trios. As a result, two different molecular pathways were found to be enriched in the different databases, namely the "Adherens junction's interactions pathway" and the "Extracellular matrix organization pathway", while a third pathway, the "NCAM1 molecular pathway" showed a trend for significance when the severity of autistic symptoms was taken into consideration. Our findings are consistent with what was anticipated to be one of the most critical molecular pathways in Autism [29]. Cell adhesion proteins are well known to influence the neuronal function. Rendall and colleagues recently reported on the activity of

\begin{tabular}{|c|c|c|c|c|c|c|c|c|}
\hline ID & Description & Gene Ratio & Bg Ratio & $P$-value & p.adjust & Q-value & Gene ID & Count \\
\hline 418990 & $\begin{array}{l}\text { Adherens junctions } \\
\text { interactions }\end{array}$ & $12 / 615$ & $31 / 6750$ & 8.20E-06 & 8.20E-03 & $8.20 \mathrm{E}-03$ & 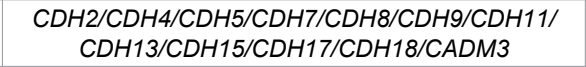 & 12 \\
\hline 422475 & Axon guidance & $45 / 615$ & $292 / 6750$ & 2.70E-04 & $1.30 \mathrm{E}-01$ & $1.30 \mathrm{E}-01$ & $\begin{array}{c}\text { AP2B1/CACNA1C/CACNB2/CFL1/CNTN1/COL4A3/ } \\
\text { COL5A1/COL6A3/NCAN/DLG1/EFNA5/EPHA3/ } \\
\text { EPHA7/FYN/GRIN2B/ITGA9/ITGB3/KCNQ3/ } \\
\text { ABLIM1/MYH11/NCAM1/PLXNA2/PRKCQ/RAF1/ } \\
\text { ROBO1/ROBO2/RPS6KA2/SH3GL2/SOS2/CNTN2/ } \\
\text { EZR/NCK2/KALRN/SEMA5A/RANBP9/ACTR3/ } \\
\text { PLXNC1/CAP2/CNTN6/TREM2/TRPC7/NTN4/ } \\
\text { SEMA6D/PLXNA4/RGMB }\end{array}$ & 45 \\
\hline 381426 & $\begin{array}{l}\text { Regulation of } \\
\text { Insulin-like Growth } \\
\text { Factor (IGF) } \\
\text { transport and } \\
\text { uptake by Insulin- } \\
\text { like Growth Factor } \\
\text { Binding Proteins } \\
\text { (IGFBPs) }\end{array}$ & $7 / 615$ & $17 / 6750$ & 4.30E-04 & $1.50 \mathrm{E}-01$ & $1.50 \mathrm{E}-01$ & F2/IGF1/IGFBP1/IGFBP3/PAPPA/PLG/PAPPA2 & 7 \\
\hline 202430 & $\begin{array}{l}\text { Translocation } \\
\text { of ZAP-70 to } \\
\text { Immunological } \\
\text { synapse }\end{array}$ & $7 / 615$ & $18 / 6750$ & $6.60 \mathrm{E}-04$ & 1.60E-01 & $1.60 \mathrm{E}-01$ & $\begin{array}{c}H L A-D Q A 1 / H L A-D Q A 2 / H L A-D Q B 1 / H L A-D Q B 2 / H L A- \\
D R A / H L A-D R B 1 / H L A-D R B 5\end{array}$ & 7 \\
\hline
\end{tabular}

Note: ID=Molecular pathways' ID; Description=Description of the pathway; GeneRatio=Number of genes in pathway in the selected database/number of genes overall in the selected database; BgRation=Number of genes in the pathway in international dataset/number of genes overall in international datasets; $p$.adjust $=p$ values after Bonferroni correction; $q$ value $=p$ values after false discovery rate correction for multiple testing . 


\begin{tabular}{|c|c|c|c|c|c|c|c|c|}
\hline ID & Description & Gene Ratio & Bg Ratio & $P$-value & p.adjust & Q-value & Gene ID & Count \\
\hline 1474244 & $\begin{array}{c}\text { Extracellular matrix } \\
\text { organization }\end{array}$ & $42 / 570$ & $249 / 6748$ & 8.82E-06 & 0.00778 & 0.0074 & $\begin{array}{c}\text { BMP1/DST/DDR1/CD44/CD47/COL4A1/COL4A4/COL5A1/ } \\
\text { COL5A2/COL7A1/COL8A1/COL9A1/COL19A1/COMP/ } \\
\text { HAPLN1/DMD/FBLN1/FBN2/EFEMP1/ITGA6/ITGAM/ITGAX/ } \\
\text { KLKB1/LAMA2/LAMA4/LTBP1/NCAM1/PLG/SPARC/COL14A1/ } \\
\text { VWF/GDF5/MMP20/NRXN1/SDC3/LAMC3/FBLN5/ADAMTS5/ } \\
\text { COL25A1/COL23A1/LAMA1/COL28A1 }\end{array}$ & 42 \\
\hline 422475 & Axon guidance & $43 / 570$ & $292 / 6748$ & 0.0001 & 0.084 & 0.081 & $\begin{array}{c}\text { ABL1/ALCAM/CACNA1C/CNTN1/COL4A1/COL4A4/COL5A1/ } \\
\text { COL5A2/COL9A1/EFNA5/EGFR/EPHA7/EPHA8/ABLIM1/ } \\
\text { MET/NCAM1/PLXNA2/PRKCQ/PTPN11/RPS6KA2/SCN1B/ } \\
\text { SOS2/SPTAN1/SPTB/SPTBN1/TRPC6/ST8SIA4/NCK2/SLIT2/ } \\
\text { RANBP9/PLXNC1/CHL1/CLASP2/PLXND1/CNTN6/DPYSL5/ } \\
\text { TRPC7/SRGAP1/SEMA6D/APH1B/ABLIM2/UNC5D/LAMA1 }\end{array}$ & 43 \\
\hline
\end{tabular}

Note: ID=molecular pathways' ID; Description=Description of the pathway; GeneRatio=Number of genes in pathway in the selected database/number of genes overall in the selected database; BgRation=Number of genes in the pathway in international dataset/number of genes overall in international datasets; $p$.adjus $t=p$ values after Bonferroni correction; $q$ value $=p$ values after false discovery rate correction for multiple testing.

Table 2: Molecular pathways analysis on Autism sample 4

the CNTNAP2, a cell adhesion protein whose deletion results into myelin formation's insufficiency and delayed learning, two possible characteristics of ASD [30]. On the other hand, cell-adhesion molecules of the immunoglobulin superfamily have a critical role in brain development and in the maintenance of synaptic plasticity, and their defect can severely alter the function of the brain [31-33]. One of the most known and investigated gene located in the "Adherens junction's interactions pathway" is the Catenin Beta 1 (CTNNB1). Its product is a part of the proteins that consitute adherens junctions and it is involved in the Wnt pathway and the Reeling Pathway. The Wnt pathway is a complex set of different molecular pathways that mostly control 1) gene expression; 2) cell polarity and 3) calcium balance. It is relevant in cell fate, cell proliferation and cell migration. Variations within the pathway lead to cancer, diabetes and at least other 100 diseases (http:// www.malacards.org/search/results/CTNNB1), which also include mental retardation. Evidence showing an involvement of the Wnt pathway in Autism is also gathering [34-36]. Quite interestingly, Wong and colleagues reported that the manipulation of the extracellular environment with pro-inflammatory mediators may impact both the activation of the Wnt molecular pathway and the activation of the metalloproteases that modify the extracellular matrix and allow and facilitate the cells' migration [37]. Metalloproteases are one of the principal enzymes of the "Extracellular matrix organization pathway". Disturbances in inflammation have been reported to be possible causes to Autism [38,39], and neurodevelopment is dependent on the efficiency of the immune system [40-42]. It is tempting to postulate, that a signal from a genetic susceptibility to an inflammation event during neurodevelopment was detected in the present contribution. A less efficient molecular cascade (the "Adherens junction's interactions pathway" or the "Extracellular matrix organization pathway") would respond in a less efficient way to inflammatory insults, this resulting in higher risk for ASD. This hypothesis needs to be further tested by independent analyses. Consistently with this, one of the genes that is included in the "Adherens junctions interactions pathway", the CADM2, was previously found to be associated with ASD [43], in a sample of 1402 trios of ASD. Another gene included in the same molecular pathway, the ACTB gene, was deleted in a single case report a child exhibiting autistic like behavior along with brachycephaly, prominent ears, cryptorchidism, speech delay, poor eye contact, and outburst of aggressive behavior. Finally, NCAM1 is also a cell-adhesion molecule whose activity impacts on a wide range of events including cellular adhesion, migration, proliferation, differentiation, survival and synaptic plasticity.

\section{Conclusion and Limitations}

Adhesion molecules have been candidates for unraveling the genetics of Autism in the last decades, but results have been inconsistent or negative. A possible explanation to this apparently conflicting finding is the poor penetrance of the single variations for the phenotype under investigation. When investigated alone, SNPs do not have sufficient power to emerge from the noise signal in the most common association analyses. This caveat is addressed by a molecular pathway analysis, where the weak signals from different SNPs are gathered by the molecular pathway labeling, acquiring power to emerge as statistical significant. Molecular pathway analysis may be a promising approach to GWAS data, but it also has limits. One of the main limits of the genome wide molecular pathway analysis is that it relies on known molecular pathways and known genetic functions. Moreover, this technique is limited by the numbers of available SNPs included in the GWAS, so that the risk of false negative findings due to poor coverage of specific genes cannot be ruled out. Keeping this in mind, it is possible to combine the results of the molecular pathway analysis with the current published evidence about a specific phenotype, helping defining the genetic makeup that increases the risk for a disease or a group of diseases. Another limit of the present contribution is that it cannot take into account the de novo variations, as they may not be labeled and cannot therefore be grouped in any molecular pathway. De novo variations may explain a part of the missing heritability, but it was estimated that $49 \%$ of the genetic architecture of ASD is related to common inherited variants and only $3 \%$ by de novo variations and rare variants [44].

\section{Acknowledgements}

\section{Datasets}

Genetic data were available from the NIMH Center for collaborative genetic studies.

NIMH Study 65, also known as AGP or TASC (PI: Gallagher), deposited genotype data in four sets, cleaned and raw, stage 1 and stage 2 . NIMH then combined the cleaned stage 1 and stage 2 data into one dataset named Autism Dataset 4 , in the PLINK file format. The $n=2893$ records in Dataset 4 occur in $n=964$ families (99\% trios), of which $n=935$ families have one or more probands with a diagnosis, ranging from strict to broad autism, see documentation for definitions. The AGP Simplex Collection (TASC) was funded by an award from Autism Speaks and by funding support to the repository development by the NIMH. The principal investigator and co-investigators on this study were Louise Gallagher, Trinity College Dublin; Astrid Vicente, Instituto Gulbenkian de Ciencia, Oeira; Joseph Buxbaum Mount Sinai School of Medicine; Peter Szatmari, McMaster University; William McMahon, University of Utah; Michael Cuccaro, University of Miami; James Sutcliffe, Vanderbilt University; Christine Freitag, Klinikum der Johann-Wolfgang GoetheUniversität, Frankfurt/Main; Sabine Klauck, Deutsches Krebsforschungszentrum (DKFZ), Heidelberg; Veronica Vieland (DCC Director), Research Institute at Nationwide Children's Hospital, Ohio; Dan Geschwind, AGRE/UCLA; John Nurnberger, University of Indiana; Ed Cook, University of Illinois at Chicago; Raphae Bernier, University of Washington/CPEA. Genotype data were generated by Dan Arking and Aravinda Chakravarti using 1,264 samples that were obtained from the NIMH Center for Collaborative Genetic Studies on Mental Disorders. This study 
Citation: Drago A, Calabro M, Crisafulli C, Rasmussen SK (2018) Genetic Insights from a Molecular Pathway Analysis on Two Independent Samples of Autistic Patients. J Mol Genet Med 12: 326 doi:10.4172/1747-0862.1000326

was supported by grants from the NIMH (R01 MH060007) to Aravinda Chakravart and Dan Arking. Arking DE, Cutler DJ, Brune CW, Teslovich TM, West K, Ikeda M Rea A, Guy M, Lin S, Cook EH, Jr., Chakravarti A. A Common Genetic Variant in the Neurexin Superfamily Member CNTNAP2 Increases Familial Risk of Autism. Am J Hum Genet 2008; 82:160-164.

\section{Computational power}

A computer cluster was used to undertake the analyses. Computation power was required for the permutation analysis. The GenomeDK HPC cluster at Aarhus University comprises of 190 nodes (3384 compute cores) connected with $10 \mathrm{GigE} /$ Infiniband. Each node has from 16 to 32 cores and either $64 \mathrm{~GB}, 128 \mathrm{~GB}, 256 \mathrm{~GB}$ 512 GB or 1 TB of RAM. The cluster has been designed specifically for bioinformatic workloads and has a storage capacity of $3.5 \mathrm{~PB}$.

\section{Conflict of Interest}

Authors declare no conflicts of interest.

\section{References}

1. Geschwind DH (2009) Advances in autism. Ann Rev Med 60: 367-380.

2. Lai MC, Lombardo MV, Baron-Cohen S (2014) Autism. Lancet 383: 896-910.

3. Ozonoff, S, Young GS, Carter A, Messinger D, Yirmiya N, et al. (2011) Recurrence risk for autism spectrum disorders: A Baby Siblings Research Consortium study. Pediatrics 128: e488-495.

4. Bailey A, Le Couteur A, Gottesman I, Bolton P, Simonoff E, et al. (1995) Autism as a strongly genetic disorder: Evidence from a British twin study. Psychol Med 25: $63-77$

5. Devlin B, Melhem N, Roeder K (2011) Do common variants play a role in risk for autism? Evidence and theoretical musings. Brain Res 1380: 78-84.

6. Adams HHH, Hibar DP, Chouraki V, Stein JL, Nyquist PA, et al. (2016) Nove genetic loci underlying human intracranial volume identified through genomewide association. Nature Neurosci 1: 1-2.

7. Torrico B, Chiocchetti AG, Bacchelli E, Trabetti E, Hervás A, et al. (2016) Lack of replication of previous autism spectrum disorder GWAS hits in European populations. Autism Res 1: 2-4

8. Yin CL, Chen HI, Li LH, Chien YL, Liao HM, et al. (2016) Genome-wide analysis of copy number variations identifies PARK2 as a candidate gene for autism spectrum disorder. Mol Autism 7: 23

9. Stessman HAF, Willemsen MH, Fenckova M, Penn O, Hoischen A, et al (2016) Disruption of POGZ is associated with intellectual disability and autism spectrum disorders. Am J Hum Genet 98: 541-552.

10. Wang Y, Zhao X, Ju W, Flory M, Zhong J, et al. (2015) Genome-wide differential expression of synaptic long noncoding RNAs in autism spectrum disorder. Transl Psych 5: e660.

11. Liu X, Shimada T, Otowa T, Wu YY, Kawamura Y, et al. (2016) Genome-wide Association Study of Autism Spectrum Disorder in the East Asian Populations. Autism Res 9: 340-349.

12. Connolly S, Anney R, Gallagher L, Heron EA (2016) A genome-wide investigation into parent-of-origin effects in autism spectrum disorder identifies previously associated genes including SHANK3. Eur J Hum Genet 1: 2-4.

13. Klei L, Sanders SJ, Murtha MT, Hus V, Lowe JK, et al. (2012) Common genetic variants, acting additively, are a major source of risk for autism. Mol Autism 3: 9

14. Geschwind DH, State MW (2015) Gene hunting in autism spectrum disorder: On the path to precision medicine. Lancet Neurol 14: 1109-1120.

15. Connolly JJ, Glessner JT, Hakonarson H (2013) A genome-wide association study of autism incorporating autism diagnostic interview-revised, autism diagnostic observation schedule, and social responsiveness scale. Child Develop 84: 17-33.

16. Chaste P, Klei L, Sanders SJ, Hus V, Murtha MT, et al. (2015) A genomewide association study of autism using the Simons Simplex Collection: Does reducing phenotypic heterogeneity in autism increase genetic homogeneity? Biol Psych 77: 775-784.

17. Berg JM, Geschwind DH (2012) Autism genetics: Searching for specificity and convergence. Genome Biol 13: 247.

18. Holmans $P$ (2010) Statistical methods for pathway analysis of genome-wide data for association with complex genetic traits. Advan Genet 72: 141-179.
19. Ebrahimi-Fakhari D, Sahin M (2015) Autism and the synapse: emerging mechanisms and mechanism-based therapies. Curr Opinion Neurol 28: 91102.

20. Sahin M, Sur M (2015) Genes, circuits, and precision therapies for autism and related neurodevelopmental disorders. Sci 1: 350-352.

21. Feldman R, Monakhov M, Pratt M, Ebstein RP (2016) Oxytocin pathway genes: Evolutionary ancient system impacting on human affiliation, sociality, and psychopathology. Biol Psych 79: 174-184.

22. Drago A, Calabrò M, Crisafulli C, Jensen BU (2016) A molecular pathway analysis on a sample trios of autistic patients. 29th ECNP Congress, Vienna, Austria.

23. Spielman RS, McGinnis RE, Ewens WJ (1993) Transmission test for linkage disequilibrium: the insulin gene region and insulin-dependent diabetes mellitus (IDDM). Am J Hum Genet 52: 506-516.

24. Purcell S, Neale B, Todd-Brown K, Thomas L, Ferreira MAR, et al. (2007) PLINK: A tool set for whole-genome association and population-based linkage analyses. Am J Hum Genet 81: 559-575.

25. R-Foundation for Statistical Computing (2013) R: A language and environment for statistical computing. R Development Core Team.

26. Huber W, Carey VJ, Gentleman R, Anders S, Carlson M, et al. (2015) Orchestrating high-throughput genomic analysis with Bioconductor. Nature Methods 12: 115-121.

27. Yu G, He QY (2016) Reactome PA: An R/Bioconductor package for reactome pathway analysis and visualization. Mol BioSys 12: 477-479.

28. Croft D, Mundo AF, Haw R, Milacic M, Weiser J, et al. (2014) The Reactome pathway knowledgebase. Nucleic Acids Res 42: D472-477.

29. Pescosolido MF, Yang U, Sabbagh M, Morrow EM (2012) Lighting a path: Genetic studies pinpoint neurodevelopmental mechanisms in autism and related disorders. Dialog Clin Neurosci 14: 239-252.

30. Rendall AR, Truong DT, Fitch RH (2016) Learning delays in a mouse model of Autism Spectrum Disorder. Behav Brain Res 303: 201-217.

31. Bhalla K, Luo Y, Buchan T, Beachem MA, Guzauskas GF, et al. (2008) Alterations in $\mathrm{CDH} 15$ and KIRREL3 in patients with mild to severe intellectual disability. Am J Hum Genet 83: 703-713.

32. Guerin A, Stavropoulos DJ, Diab Y, Chénier S, Christensen $\mathrm{H}$, et al. (2012) Interstitial deletion of 11q-implicating the KIRREL3 gene in the neurocognitive delay associated with Jacobsen syndrome. Am J Med Genet Part A 158A: 2551-2556.

33. Liu YF, Sowell SM, Luo Y, Chaubey A, Cameron RS, et al. (2015) Autism and Intellectual Disability-Associated KIRREL3 Interacts with Neuronal Proteins MAP1B and MYO16 with Potential Roles in Neurodevelopment. PloS One 10: e0123106.

34. Kalkman HO (2012) A review of the evidence for the canonical Wnt pathway in autism spectrum disorders. Mol Autism 3: 10.

35. Caracci MO, Ávila ME, De Ferrari GV (2016) Synaptic Wnt/GSK3 $\beta$ Signaling Hub in Autism. Neural Plasticity 2016: 9603751.

36. Packer A (2016) Enrichment of factors regulating canonical Wnt signaling among autism risk genes. Mol Psych 1: 1-2.

37. Wong CT, Ahmad E, Li H, Crawford DA (2014) Prostaglandin E2 alters Wnt-dependent migration and proliferation in neuroectodermal stem cells: Implications for autism spectrum disorders. Cell Commun Signal 12: 19.

38. Ornoy A, Weinstein-Fudim L, Ergaz Z (2015) Prenatal factors associated with autism spectrum disorder (ASD). Reprod Toxicol 56: 155-169.

39. Wetie AG, Wormwood KL, Charette L, Ryan JP, Woods AG, et al. (2015) Comparative two-dimensional polyacrylamide gel electrophoresis of the salivary proteome of children with autism spectrum disorder. J Cellular Mo Med 19: 2664-2678.

40. Mead J, Ashwood P (2015) Evidence supporting an altered immune response in ASD. Immunol Letters 163: 49-55.

41. Filiano AJ, Gadani SP, Kipnis J (2015) Interactions of innate and adaptive immunity in brain development and function. Brain Res 1617: 18-27.

42. Ashwood P, Wills S, Van de Water J (2006) The immune response in autism: a new frontier for autism research. J Leuko Biol 80: 1-15. 
Citation: Drago A, Calabro M, Crisafulli C, Rasmussen SK (2018) Genetic Insights from a Molecular Pathway Analysis on Two Independent Samples of Autistic Patients. J Mol Genet Med 12: 326 doi:10.4172/1747-0862.1000326

Page 5 of 5

43. Casey JP, Magalhaes T, Conroy JM, Regan R, Shah N, et al. (2012) A novel approach of homozygous haplotype sharing identifies candidate genes in autism spectrum disorder. Hum Genet 131: 565-579.
44. Gaugler T, Klei L, Sanders SJ, Bodea CA, Goldberg AP, et al. (2014) Most genetic risk for autism resides with common variation. Nature Genet 46: $881-$ 885 